\title{
FLAIR, a Facility for Low-energy Antiproton and Ion Research
}

\author{
Eberhard Widmann \\ Stefan Meyer Institut für subatomare Physik \\ Austrian Academy of Sciences \\ Boltzmanngasse 3, A-1090 Wien, Austria
}

\begin{abstract}
The FAIR facility for beams of ions and antiprotons at Darmstadt will provide antiproton beams of intensities that are two orders of magnitude higher than currently available. The low-energy antiproton physics community has recognized the opportunity to create a next-generation low-energy antiproton facility and has submitted a proposal for a facility called FLAIR which was very positively evaluated in spring of 2005. FLAIR will be able to provide cooled antiproton beams well below $100 \mathrm{keV}$ kinetic energy, which will allow for a much higher rate of trapped antiprotons and therefore greatly advance the currently performed experiments. Furthermore, the availability of continuous beams will make many new experiments possible. This talk gives an overview of the layout and physics program of the proposed facility.
\end{abstract}

\section{Introduction}

Low-energy antiproton physics is currently being done at the Antiproton Decelerator (AD) of CERN, Geneva. Due to the low intensity $\left(\sim 10^{5} \overline{\mathrm{p}} / \mathrm{s}\right)$ and the availability of only pulsed extraction, the physics program is limited to the spectroscopy of antiprotonic atoms and antihydrogen formed in charged particle traps or by stopping antiprotons in low-density gas targets (see talks by W. Oelert, R.S. Hayano, G. Bonomi, and G. Gabrielse at this conference). Furthermore, the output energy of the $\mathrm{AD}$ (5 $\mathrm{MeV}$ kinetic energy) is still significantly higher than the $<100 \mathrm{keV}$ energy best suited for these experiments.

At FAIR, the planned Facility for Antiproton and Ion Research at Darmstadt, it will become possible to create a next-generation low-energy antiproton facility to overcome these limitations by providing cooled beams at higher intensities and a factor 100 lower energy. In addition the new facility should have the possibility of slow (i. e. continuous) extraction, which will allow nuclear/particle physics type experiments requiring coincidence measurements to be performed. 
In 2004 a letter of intent [1] and in 2005 a technical proposal [2] have been submitted to GSI, Darmstadt, for a facility called FLAIR (Facility for Low-energy Antiproton and Ion Research) that is described in the following. Both documents have been evaluated very positively by the program advisory committee of GSI. FLAIR consists of two storage rings, a magnetic (LSR) and an electrostatic (USR) one, and a universal trap facility (HITRAP), cf. Fig. 1. These components of the facility can provide stored as well as fast and slow extracted cooled beams at energies between $30 \mathrm{MeV}$ and $300 \mathrm{keV}$ (LSR), between $300 \mathrm{keV}$ and $20 \mathrm{keV}$ (USR), and cooled particles at rest or at ultra-low $(\mathrm{eV}-\mathrm{keV})$ energies (HITRAP). This will allow a large variety of new experiments to be performed, as described in Sec. 3. Among the unique experiments only possible at such a facility are nuclear physics studies using antiprotons as a hadronic probe to investigate the structure of nuclei, including radioactive isotopes produced at the future facility, and many atomic-collision type experiments with internal targets in both storage rings with effective intensities as large as $10^{10} \overline{\mathrm{p}} / \mathrm{s}$. An important synergetic aspect is that the whole structure will also be used to study highly charged ions, including storing, cooling (LSR, USR) and trapping them in Penning traps like HITRAP and investigating them in a dedicated area for heavy ions. These experiments are part of the physics program of the SPARC collaboration [3] (see talk by R. Schuch).

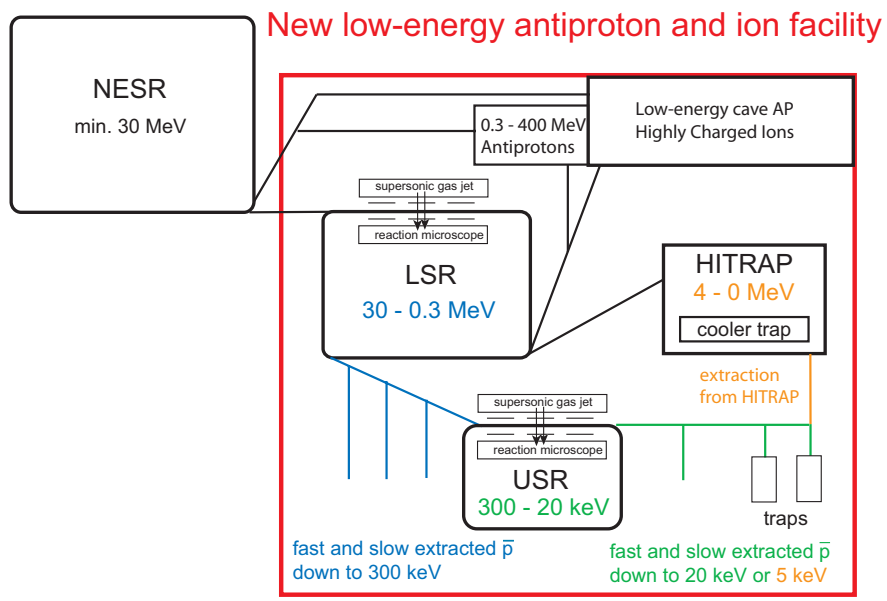

Figure 1: Layout of the low-energy antiproton and ion facility. NESR: new experimental storage ring (part of the approved FAIR project). LSR: intermediate storage ring which decelerates $\overline{\mathrm{p}}$ to $300 \mathrm{keV}$. USR: electrostatic ultra-low energy storage ring. Both rings use electron cooling to provide low-emittance beams and include internal gas jet targets for atomic collision studies. HITRAP: trap facility for efficient deceleration and cooling of $\overline{\mathrm{ps}}$ from $4 \mathrm{MeV}$ to rest. 


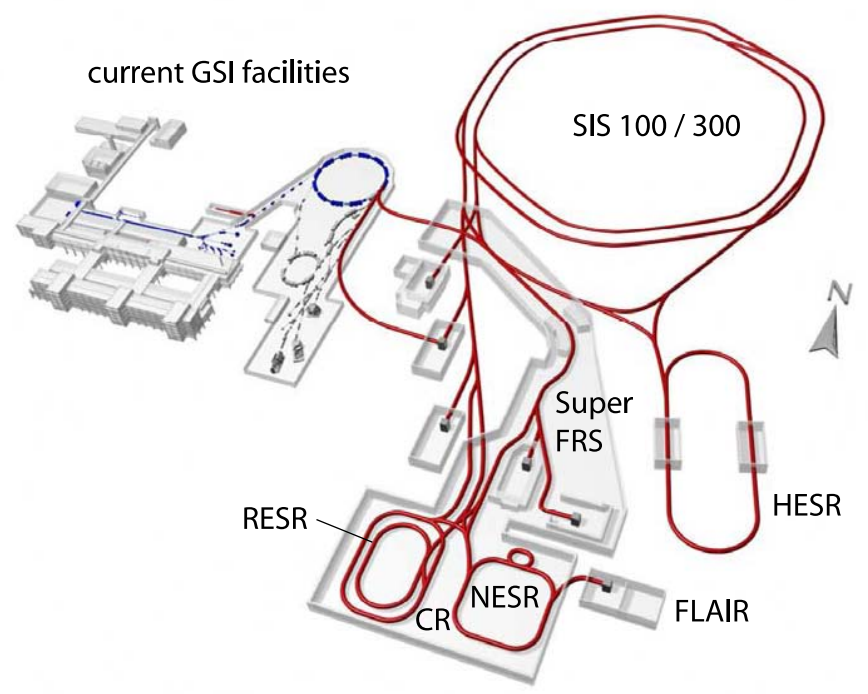

Figure 2: Overview of the FAIR facility. Left the existing GSI facility is shown, and right the newly planned facilities. Bold lines symbolize storage rings and beam pipes. SIS100/300: heavy ion synchrotron with 100/300 Tm magnetic rigidity. SuperFRS: Super fragment separator for studying short-lived nuclei. CR: capture ring for antiprotons. RESR: accumulation ring for antiprotons. HESR: highenergy storage ring for antiprotons. NESR: new experimental storage ring for experiments with highly charged ions and to decelerate antiprotons. FLAIR marks the position of the planned FLAIR facility.

\section{Layout and performance of the facility}

The key features of the proposed facility at Darmstadt (cf. Fig. 1) are:

- High-brightness, high-intensity, low-energy antiproton beams.

- High antiproton intensity due to accumulation.

- Cooled $\overline{\mathrm{p}}$ beams down to $300 \mathrm{keV}$ using LSR.

- Electrostatic storage ring (USR) for atomic collision experiments and deceleration and cooling to $20 \mathrm{keV}$.

- HITRAP for efficient deceleration of $\overline{\mathrm{ps}}$ from $4 \mathrm{MeV}$ to rest and extraction of $\overline{\mathrm{p}} \mathrm{s}$ from a cooler trap at $\mathbf{k e V}$ energies.

- Both slow and fast extraction from LSR and USR at energies between $30 \mathrm{MeV}$ and $20 \mathrm{keV}$. 
The antiproton production method at the future facility [4] (see talk by C. Schwarz) is similar to the scheme used for LEAR at CERN. $10^{8}$ antiprotons will be produced every 5 seconds, will be collected in the CR storage ring (cf. Fig. 2) and accumulated in the RESR. Already within the foreseen scheme of FAIR it is possible to decelerate antiprotons to $30 \mathrm{MeV}$ in the NESR. In order to produce cooled beams below $500 \mathrm{keV}$ starting from the lowest energy available from the NESR, the most promising scheme is to use a storage ring (low-energy storage ring, LSR). This was first proposed at CERN in the 1980's (called ELENA [5]). The energies and cooling requirements fit exactly to existing small storage rings like CRYRING (MSL, Stockholm; energy range $96 \mathrm{MeV}-300 \mathrm{keV}$ for $\overline{\mathrm{p}}$ ) or TSR (MPI-K Heidelberg), so that there is no question about the technical feasibility. CRYRING will stop operation within a few years and it is proposed to move it to FLAIR to be used as LSR. For further deceleration, an electrostatic storage ring (USR) from 300 to $20 \mathrm{keV}$ with electron cooling is being proposed by the Max Plack Institute for Nuclear Physics in Heidelberg. Antiprotons from the LSR can be trapped in the HITRAP facility [6] which is being constructed for use at the current ESR of GSI. In addition to studies with stopped antiproton, it is also foreseen to extract $\bar{p}$ at sub-keV energies.

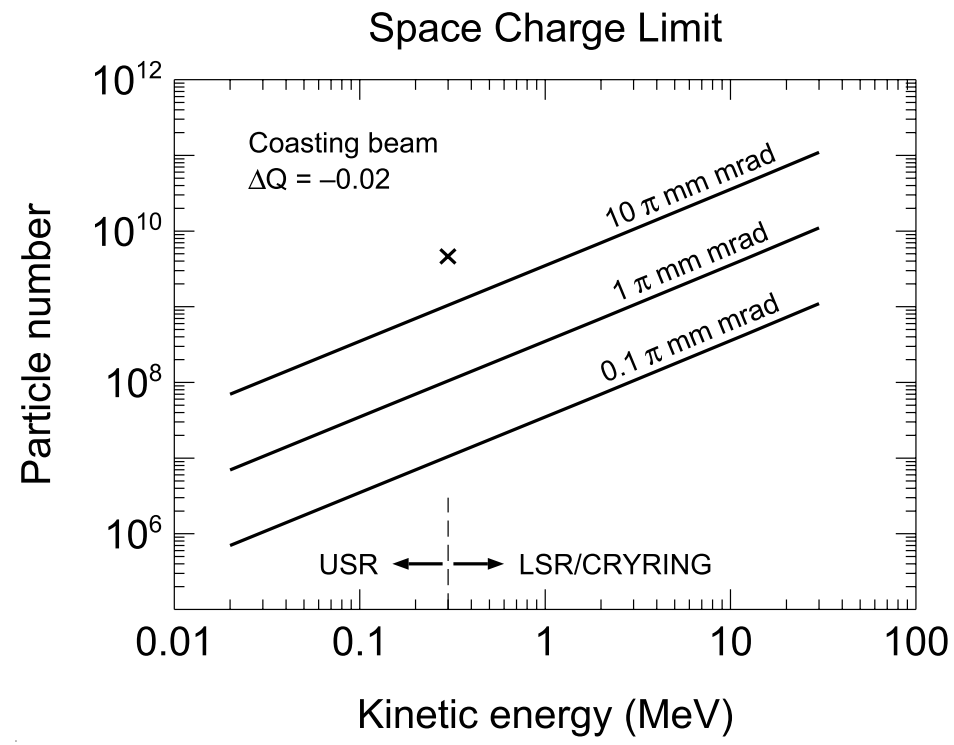

Figure 3: Maximum number of particles available in a storage ring for different emmittances due to the space charge $[2,7]$. The values are estimated for a maximum Laslett tune-shift of $\Delta Q=-0.02$, which is a measure for the distortion of particle orbits due to the space charge. The cross corresponds to a recent measurement at CRYRING. 
Fig. 3 shows the numbers of antiprotons that can be reached in the FLAIR storage rings due to the space charge limit a low energies. From this, antiproton rates of $\sim 10^{6} / \mathrm{s}$ extracted both fast and slow at energies down to $20 \mathrm{keV}$ will be available. This corresponds to about $10 \%$ of the production rate at FAIR, so that FLAIR can operate in parallel to the HESR storage ring. Antiprotons can be extracted either slow or fast from the LSR or USR and used in several beamlines for either directly stopping them in low-density gas targets using thin windows, or trapping them with high efficiency in charged particle traps. The same rate of antiprotons can be obtained at rest in HITRAP or extracted at ultra-low $(\mathrm{eV}-\mathrm{keV})$ energies. The USR will be used for deceleration, slow and fast extraction, and for atomic physics experiments with internal targets. The energy range of $300-20 \mathrm{keV}$ (using electron cooling) makes it a unique tool for many atomic collision experiments which are only possible in such a low-energy storage ring, where effective intensities ( $i$. e e the number of stored particles $N_{\text {stored }}$ times the revolution frequency $f_{\text {rev }}$, $\left.R_{\text {eff }}=N_{\text {stored }} f_{\text {rev }}\right)$ of $R_{\text {eff }}=10^{10} \overline{\mathrm{p}} / \mathrm{s}$ are reached for in-ring experiments.

The FLAIR scheme gives about a factor 100 more antiprotons per unit time stopped in gas targets or trapped in ion traps as compared to the present AD at CERN where no dedicated accumulation and multi-stage deceleration rings are utilized. The availability of such beams will tremendously increase the number of experiments possible at this facility.

\section{Physics program of FLAIR}

The physics of FLAIR covers a wide range in atomic, nuclear and particle physics and has potential medical applications. It is described in detail in the FLAIR letter of intent [1] available from the FLAIR web page. In the following a brief overview will be given.

\subsection{Precision spectroscopy of antiprotonic atoms and antihydrogen}

This is the current topic of the Antiproton Decelerator (AD) at CERN. The main goal here is to study fundamental symmetries and interactions by providing high-precision data of particle and antiparticle properties for tests of CPT symmetry (see talk by N. Mavromotos) and QED calculations. Antiprotonic atoms have been used for some time to test CPT symmetry between proton and antiproton properties (for a review, see [8]). The most accurate test of proton/antiproton properties is the measurement of their cyclotron frequency $\omega_{c} \propto Q / M(Q, M$ denoting charge and mass) by the TRAP collaboration at LEAR, yielding an accuracy of better than $10^{-10}[9]$. Separate CPT limits on $Q$ and $M$ can be set by combining this measurement with the recent precision laser spectroscopy of antiprotonic helium by the ASACUSA collaboration at the Antiproton Decelerator (AD) of CERN, which are now at a level of $10^{-8}$ (see talk by R.S. Hayano) [10]. These latter experiment also constitute a sensitive test for three-body QED calculations. Triggered by the spectroscopy results, the accuracy of three-body bound-state QED calculations has been tremendously improved, reaching a level comparable to the experimental resolution (cf. talk by V. Korobov) [11, 12]. The major recent experimental improvement came from using a radio frequency quadrupole decelerator (RFQD) to decelerate $\overline{\mathrm{p}}$ from $5 \mathrm{MeV}$ to $\sim 100 \mathrm{keV}$ which allowed to stop them in more dilute gases. Further improvements can 
be expected at FLAIR from the high-quality cooled antiprotons beams at this energy or below.

The ultimately highest precision of a CPT test with antiprotonic atoms is likely to be achieved using antihydrogen. The production of large amounts of cold antihydrogen at the AD has been reported in 2002 by both the ATHENA and ATRAP collaborations at the $\mathrm{AD}$ (cf. talks by W. Oelert, G. Bonimi, and G. Gabrielse) [13, 14, 15, 16], but it is still expected to take several years until precision spectroscopy can be performed. After a shutdown in 2005, the $\mathrm{AD}$ is expected to run until 2010, so that initial results on spectroscopy can be expected at the AD. The ultimate goal is to measure the $1 \mathrm{~S}-$ $2 \mathrm{~S}$ two-photon laser transition $[17,18]$ and the ground state hyperfine splitting [19] to accuracies similar to the ones achieved for hydrogen $\left(10^{-14}[20]\right.$ and $10^{-12}[21,22]$, resp.), yielding a very sensitive test of the CPT theorem. New ideas like the use of a cusp trap for $\overline{\mathrm{H}}$ formation [23] are being proposed. To achieve the ultimate precision, the trapping and laser cooling of neutral antihydrogen atoms is required. The development of these techniques will surely take many more years to accomplish.

Once trapped and laser-cooled antihydrogen is available, other challenging experiments can be performed. Among them is the gravitation of antimatter [24], which is a long standing question that has never been answered experimentally, because in the case of charged particles, gravitational effects are covered by the many orders of magnitude stronger electromagnetic interaction. Collisions between antihydrogen and matter atoms as well as the creation of larger antimatter systems like $\overline{\mathrm{H}}^{+}$(one antiproton and two positrons, equivalent to the well known $\mathrm{H}^{-}$ion) are of big interest for atomic collision theory.

\subsection{Atomic collision physics}

This field will greatly benefit from the availability of ultra-slow, cooled antiproton beams in storage rings. This will enable for the first time ever the detailed study of ionization processes with antiprotons in kinematically complete experiments. The energy loss can be investigated at ultra-low energies to answer open questions about the velocity dependence in this regime. Antiprotons are best suited for such studies, because unlike protons their charge is not screened by electrons which makes the theoretical treatment very difficult. The very short interaction time of less than femtoseconds for $\bar{p}$ energies above $1 \mathrm{keV}$ makes antiprotons a perfect and unique tool to study many-electron dynamics in the strongly correlated, non-linear, sub-femtosecond time regime, the most interesting and, at the same time, most challenging domain for theory.

\subsection{Antiprotons as hadronic probes}

In nuclear physics, the antiproton is used as a hadronic probe to study the nuclear structure. X-ray spectroscopy of the low-lying states of $\bar{p} p$ or other light atoms [25] gives important information on the nucleon-antinucleon interaction in the low-energy limit, where scattering experiments cannot provide precise values. These data are vital for the improvement of QCD calculations in the low-energy (hence non-perturbative) region. Xray spectroscopy of heavy antiprotonic atoms can be used to obtain information about the density ratio of neutron and protons at the nuclear periphery, $i$. e. to investigate neutron 
halo or skin effects. The PS209 experiment at LEAR has in this way provided benchmark data for nuclear structure calculations over a wide range of nuclei (cf. talk by A. Trzcinska ) [26]. This technique is much more sensitive than others like total absorption cross section measurements, and further systematic measurements with stable isotope targets will provide a more complete and systematic picture of the nuclear surface. Since halo effects are expected to be more pronounced in nuclei with a large neutron excess which are unstable, the application of this technique to unstable radioactive ions [27] available at FLAIR via the SuperFRS will generate important contributions to the study of the structure of nuclei far from stability.

In the particle physics domain, the elementary antinucleon-nucleon interaction processes still present a few unclear aspects, in spite of the wealth of physics results produced at LEAR. While many data points exist for $\overline{\mathcal{N}} \mathcal{N}$ scattering at higher momentum, there are few data close to threshold. Here, some anomalous effects have been observed (see talk by Th. Walcher) e. g. in the $\bar{n}$ p elastic cross section [28] or the indication of a resonance in the decay $J / \Psi \rightarrow \overline{\mathrm{p}} \gamma \gamma$ observed at BES [29] which are likely related to the interplay between quark and antiquark degrees of freedom. Furthermore, there exist unexplained behaviour in the systematics of the $\overline{\mathcal{N}}$-Nucleon annihilation cross section at low energies [30].

The study of baryon-baryon interactions as a basic tool for investigations of the strong interaction can be extended to the hyperon sector, where much less data exist than in the nucleon sector. Especially few data exist on strangeness $S=-2$ systems. Stopped antiprotons are very efficient for the production of $\mathrm{S}=-2$ systems via the double strangeness and charge exchange reaction $\left(\bar{K}^{*}, K\right)$ [31]. With a sizeable branching ratio the annihilation of antiprotons results in the production of a $\bar{K}^{*}$ "beam" which interacts with another nucleon via $\bar{K}^{*} N \rightarrow K \Xi$. The momenta of the $\bar{K}^{*}$ are well matched for the production of slow $\Xi$ particles which undergo efficient $\Xi N$ interactions. The proposed studies will result in detailed information of $\mathrm{S}=-2$ baryonic and possible dibaryonic states.

\subsection{Medical applications}

Recently, interest has been shown in the medical application of antiprotons for tumor therapy. This comes from the fact the antiprotons, in addition to depositing energy via their energy loss like other charged particles, annihilate when stopped in material. The annihilation produces residual nuclear fragments of high charge and low energy, which deposit a large biological dose in the immediate surrounding of the $\overline{\mathrm{p}}$ stopping distribution. Since the cooled low-emittance antiproton beams can be stopped in a welldefined region, the presumably large energy deposited locally makes them a suitable tool for tumor therapy. A test experiment is under way at the AD of CERN [32] and, if this effect is confirmed, the method can be extended at FLAIR where the high-energy antiproton beams $(50-300 \mathrm{MeV})$ needed to penetrate deep enough into human tissue are available directly from the NESR. 


\section{Conclusions}

FLAIR will be a unique next-generation low-energy antiproton and ion facility. Cooled antiprotons down to $20 \mathrm{keV}$ both in storage rings and extracted will revolutionize lowenergy antiproton physics. Continuously extracted beams at these energies will enable nuclear and particle physics type experiments currently not possible at the AD of CERN. The availability of short-lived exotic nuclei at the future facility at Darmstadt creates synergies by using antiprotons as hadronic probes for nuclear structure. Using the same facility, atomic physics experiments with highly charged ion will be possible both in beams and at rest in HITRAP.

\section{Acknowledgements}

I would like to thank the members of the FLAIR steering committee and community who contributed to the combined effort to write the letter of intent and technical proposal and who are working towards the creation of this new facility.

\section{References}

[1] FLAIR - a facility for low-energy antiproton and ion research, Letter of intent, Feb. 2004, available from http://www.oeaw.ac.at/smi/flair/.

[2] FLAIR - a facility for low-energy antiproton and ion research, Technical proposal, Jan. 2005, available from http://www.oeaw.ac.at/smi/flair/.

[3] SPARC collaboration, Stored particle atomic research collaboration, Letter of intent, 2004, available from http://www.gsi.de/sparc.

[4] An international accelerator facility for beams of ions and antiprotons, Conceptual Design Report, GSI, Nov. 2001.

[5] H. Herr, A small decelerator ring for extra low energy antiprotons (ELENA), in Ettore Majorana Int. Sci. Ser., Phys. Sci.: 17, pages 633-642, 1984.

[6] HITRAP Technical Design Report, GSI, 2003, http://www.gsi.de/documents/DOC2003-Dec-69-2.pdf.

[7] H. Danared, 2004, private communication.

[8] J. Eades and F. J. Hartmann, Rev. Mod. Phys. 71 (1999) 373.

[9] G. Gabrielse, A. Khabbaz, D. S. Hall, C. Heimann, H. Kalinowsky, and W. Jhe, Phys. Rev. Lett. 82 (1999) 3198.

[10] M. Hori, J. Eades, R. S. Hayano, T. Ishikawa, W. Pirkl, E. Widmann, H. Yamaguchi, H. A. Torii, B. Juhász, D. Horváth, and T. Yamazaki, Phys. Rev. Lett. 91 (2003) 123401.

[11] V. I. Korobov, Phys. Rev. Lett. 67 (2003) 062501, Erratum Phys. Rev. A 68, 019902.

[12] Y. Kino, N. Yamanaka, M. Kamimura, and H. Kudo, Hyp. Int. 146-147 (2003) 331.

[13] M. Amoretti, C. Amsler, G. Bonomi, A. Bouchta, P. Bowe, C. Carraro, C. L. Cesar, M. Charlton, M. J. T. Collier, M. Doser, V. Filippini, K. S. Fine, A. Fontana, M. C. Fujiwara, R. Funakoshi, P. Genova, J. S. Hangst, R. S. Hayano, M. H. Holzscheiter, 
L. V. Jrgensen, V. Lagomarsino, R. Landua, D. Lindelf, E. L. Rizzini, M. Macr, N. Madsen, G. Manuzio, M. Marchesotti, P. Montagna, H. Pruys, C. Regenfus, P. Riedler, J. Rochet, A. Rotondi, G. Rouleau, G. Testera, A. Variola, T. L. Watson, and D. P. Van Der Werf, Nature 419 (2002) 456.

[14] G. Gabrielse, N. S. Bowden, P. Oxley, A. Speck, C. H. Storry, J. N. Tan, M. Wessels, D. Grzonka, W. Oelert, G. Schepers, T. Sefzick, J. Walz, H. Pittner, T. W. Hänsch, and E. A. Hessels, Phys. Rev. Lett. 89 (2002) 213401.

[15] G. Gabrielse, N. S. Bowden, P. Oxley, A. Speck, C. H. Storry, J. N. Tan, M. Wessels, D. Grzonka, W. Oelert, G. Schepers, T. Sefzick, J. Walz, H. Pittner, T. W. Hänsch, and E. A. Hessels, Phys. Rev. Lett. 89 (2002) 233401.

[16] G. Gabrielse, Adv. At. Mol. Opt. Phys. 50 (2005).

[17] ATHENA collaboration, Antihydrogen production and precision experiments, Proposal CERN/SPSLC 96-47 / P306, 1996.

[18] ATRAP collaboration, The production and study of cold antihydrogen, Proposal CERN/SPSC 97-8 / P302, 1997.

[19] E. Widmann, J. Eades, R. S. Hayano, T. Ishikawa, W. Pirkl, M. Hori, Y. Yamazaki, A. Mohri, T. Yamazaki, D. Horváth, B. Juhász, and E. Takács, Measurement of the antihydrogen hyperfine structure, Letter of Intent CERN-SPSC-2003-009, CERN, Geneva, Switzerland, 2003.

[20] M. Niering et al., Phys. Rev. Lett. 84 (2000) 5496.

[21] H. Hellwig, R. F. C. Vessot, M. W. Levine, P. W. Zitzewitz, D. Allan, and D. J. Glaze, IEEE Trans. IM 19 (1970) 200.

[22] L. Essen, R. W. Donaldson, M. J. Bangham, and E. G. Hope, Nature 229 (1971) 110 .

[23] A. Mohri and Y. Yamazaki, Europhys. Lett. 63 (2003) 207.

[24] J. Walz and T. W. Hänsch, General Relativity and Gravitation 36 (2004) 561.

[25] D. Gotta, Progr. Part. Nucl. Phys. 52 (2004) 133.

[26] A. Trzcińska, J. Jastrzȩbski, P. Lubiński, F. Hartmann, R. Schmidt, T. von Egidy, and B. Kłos, Phys. Rev. Lett. 87 (2001) 082501.

[27] M. Wada and Y. Yamazaki, Nucl. Instr. Meth. B 214 (2004) 196.

[28] A. Filippi, Nucl. Instr. Meth. B 214 (2004) 65.

[29] J. Z. Bai et al., Phys. Rev. Lett. 91 (2003) 022001.

[30] A. Bianconi, G. Bonomi, M. P. Bussa, E. L. Rizzini, L. Venturelli, and A. Zenoni, Phys. Lett. B 483 (2000) 353.

[31] K. Kilian, in Proc. 4th LEAR Workshop, Villars-sur-Ollon 1987, edited by C. Amsler et al., page 529, Harwood Academic Publishers, Chur, 1988, K. Kilian et al., Memorandum PSCC CERN 1990.

[32] Relative biological effectiveness and peripheral damage of antiproton annihilation, proposal CERN-SPSC-2002-030; SPSC-P-324, CERN, Geneva, Switzerland, 2002. 
